EXTENDED REPORT

\title{
Retinoblastoma patients with high risk ocular pathological features: who needs adjuvant therapy?
}

\author{
G L Chantada, I J Dunkel, M T G de Dávila, D H Abramson
}

Br J Ophthalmol 2004;88:1069-1073. doi: 10.1136/bjo.2003.037044

See end of article for authors' affiliations ......................

Correspondence to: Dr G L Chantada, Hematologia/Oncologia, Hospital JP Garrahan, Combate de los Pozos 1881, C1245AAL Buenos Aires, Argentina; gchantada@ garrahan.gov.ar

Accepted 1 January 2004

\begin{abstract}
Aims: To describe the outcome of patients with non-metastatic unilateral retinoblastoma with high risk histopathological features after primary enucleation, and to clarify the need and results of adjuvant therapy.

Patients and methods: From 1980 to 2001 adjuvant therapy was recommended only to patients with scleral involvement, post-laminar optic nerve involvement (PLONI) with either a positive margin or associated choroidal involvement, or (before 1994) isolated PLONI.

Results: 108 of 224 patients had at least one high risk feature (choroidal, scleral, anterior chamber, and/ or PLONI). Patients with isolated choroidal $(n=55)$ or anterior chamber $(n=2)$ invasion, and most with PLONI without other risk factors ( $n=21$ ) were not treated; three relapsed but are long term survivors after intensive therapy. Four with isolated PLONI received adjuvant chemotherapy and none relapsed. Three of 11 with PLONI and concomitant choroidal or scleral involvement who received adjuvant therapy relapsed, versus two of four not treated. Two of five with scleral disease relapsed. All 12 with cut end involvement received adjuvant treatment and none relapsed. In the total group, all four patients who relapsed after adjuvant therapy died.

Conclusions: Relapsing patients can be rescued with intensive therapy. Those with isolated choroidal or PLONI have a good prognosis without adjuvant therapy. Patients with PLONI with a positive margin have a good prognosis if treated with combined therapy. Those with scleral involvement or PLONI with concomitant choroid disease may benefit from adjuvant therapy.
\end{abstract}

E ven though the event free survival for patients with nonmetastatic retinoblastoma is about $95 \%$ in industrialised countries, some patients suffer extraocular relapses that may lead to death, especially when the central nervous system (CNS) is involved. Several factors thought to be associated with a high risk for extraocular recurrence have been identified upon histopathological examination of enucleated eyes, including invasion of the post-laminar optic nerve, choroid, sclera and in some studies, the anterior chamber. ${ }^{1-5}$

The use of post-enucleation adjuvant therapy in order to decrease the extraocular relapse rate in patients with high risk features is frequently considered; however, most evidence comes from relatively small prospective studies or retrospective analyses with considerable variation in inclusion criteria and treatment philosophy. ${ }^{1-36}$ There are no published randomised trials addressing the value of adjuvant therapy in this selected population which would require large international cooperative studies.

As extraocular relapse is considered by some groups a catastrophic event associated with high mortality, the aim of adjuvant therapy is to reduce its occurrence in patients with putative high risk features. ${ }^{3}$ However, the ultimate outcome of patients with extraocular relapse when treated intensively with current multimodality therapy has improved in recent years. ${ }^{7-9}$

Because of the scant evidence published on this subject, there is much controversy about the indications for adjuvant therapy, which agents should be included, and whether adjuvant therapy actually improves outcome in patients with high risk disease. The current treatment philosophies vary among centres and include recommending adjuvant treatment to all patients with known histopathological risk factors in an attempt to prevent extraocular relapse. ${ }^{3}$ This philosophy potentially exposes many children to unnecessary treatment with its inherent risks and cost, with the aim of benefiting a few. We pursued an alternative strategy: prescribing adjuvant treatment only to those hypothesised to be at the very highest risk, and treating intensively the few patients without those features who relapse.

Since its opening in 1987, the Hospital JP Garrahan (HPG) has used the clinicopathological classification and treatment recommendations of the New York Presbyterian Hospital (NYPH) for retinoblastoma, ${ }^{6}{ }^{10}$ so the combined analysis of both institutions described in this publication includes a large patient population treated under very similar guidelines and provides important information about this controversial issue.

\section{PATIENTS AND METHODS}

For this retrospective study, the medical records of all patients with unilateral retinoblastoma treated with primary enucleation from January 1980 to December 2001 at the NYPH and from August 1987 to December 2001 at the HPG were reviewed. By selecting only enucleated unilateral patients it was possible to define more precisely the impact of histopathological risk factors on outcome, as confounding variables potentially present in bilateral patients—such as systemic treatment for the fellow eye-are not present. Patients with overt extraocular disease at diagnosis and those not treated with initial enucleation at the participating institutions were excluded. Demographic information, clinical and outcome data, and pathological reports were retrieved from the computerised databases of both institutions and supplemented with chart review. All pathology

Abbreviations: CNS, central nervous system; HPG, Hospital JP Garrahan; NYPH, New York Presbyterian Hospital; pOS, probability of overall survival; pEFS, probability of event free survival. 
specimens at the HPG were reviewed by the same pathologist (MTGD) for stage assignment for two prospective studies.

At diagnosis, all patients underwent full ophthalmological examination under anaesthesia and CT scan or MRI of the head including the orbit. Lumbar puncture with cell count and examination of the cytocentrifugate (in 50\% alcohol) and bilateral bone marrow aspiration and biopsy were done in most patients before 1994 and were recommended when the pathology report revealed potentially high risk features thereafter.

Similar criteria for recommending adjuvant therapy were followed in both institutions. Patients with stage I (according to Grabowski-Abramson classification ${ }^{10}$ (intraretinal disease Ia, prelaminar optic nerve Ib, anterior chamber and isolated choroidal invasion of any degree Ic) did not receive adjuvant therapy. Patients with post-laminar optic nerve involvement with tumour at the line of section (positive margin) (stage IIb2) received adjuvant chemotherapy and orbital external beam radiotherapy (40-45 Gy). Chemotherapy was recommended for patients with microscopic scleral involvement (stage IIal). Most patients with post-laminar optic nerve invasion without involvement of the cut end (negative margin) (stage IIbl) were enrolled in two consecutive prospective studies at the HPG. Those included in the first study (1987-1993), received adjuvant chemotherapy including intrathecal chemotherapy. ${ }^{6}$ In the second study (19942001 ), only those patients with concomitant scleral or major choroidal invasion received adjuvant chemotherapy. Major choroidal invasion was defined by the presence of more than one clump of tumour cells and/or major width invasion of the choroid involving more than its half thickness. Those without these associated features did not receive adjuvant therapy. Patients with post-laminar optic nerve without invasion of the cut end were treated at the NYPH under similar guidelines. Those diagnosed up to 1994 received chemotherapy (regimen 1 in table 1); however, after 1994 they received no adjuvant therapy regardless of choroidal or scleral invasion.

Table 1 Description of chemotherapy regimens used

\begin{tabular}{l}
\hline Regimen 1 \\
\hline Week 0: cyclophosphamide: $40 \mathrm{mg} / \mathrm{kg}$ (day 1), doxorubicin $0.67 \mathrm{mg}$ / \\
$\mathrm{kg}$ (days 1-3), vincristine: $0.05 \mathrm{mg} / \mathrm{kg}$ (day 1). \\
Weeks $3,6,9,12,15,18,21:$ cyclophosphamide: $20 \mathrm{mg} / \mathrm{kg}$ (day 1), \\
doxorubicin $0.67 \mathrm{mg} / \mathrm{kg}$ (days 1 to 3 ), vincristine: $0.05 \mathrm{mg} / \mathrm{kg}$ (day 1). \\
Weeks $24,27,30,33,36,39,42,45,48,51,54:$ cyclophosphamide: \\
$30 \mathrm{mg} / \mathrm{kg}$ (day 1), vincristine: $0.05 \mathrm{mg} / \mathrm{kg}$ (day 1). \\
Intrathecal chemotherapy was given on weeks $0,1,2,3,4$, and 5. \\
\hline Regimen 2
\end{tabular}

Weeks 0, 6, 12, 18: cyclophosphamide: $40 \mathrm{mg} / \mathrm{kg}$ (day 1), MESNA: $40 \mathrm{mg} / \mathrm{kg}$ (day 1), vincristine: $0.05 \mathrm{mg} / \mathrm{kg}$ (day 1 ).

Weeks $3,9,15,21$ : carboplatin $18.7 \mathrm{mg} / \mathrm{kg}$ (patients less than $10 \mathrm{~kg}$ ) or $560 \mathrm{mg} / \mathrm{m}^{2}$ (more than $10 \mathrm{~kg}$ ) (day 1), etoposide $3.3 \mathrm{mg} / \mathrm{kg}$ (less than $10 \mathrm{~kg}$ ) or $100 \mathrm{mg} / \mathrm{m}^{2}$ (more than $10 \mathrm{~kg}$ ) (days 1 and 2).

Regimen 3

Weeks 0, 6, 12, 18: cyclophosphamide: $65 \mathrm{mg} / \mathrm{kg}$ (day 1), MESNA: $60 \mathrm{mg} / \mathrm{kg}$ (day 1), vincristine: $0.05 \mathrm{mg} / \mathrm{kg}$ (day 1), idarubicin $10 \mathrm{mg}$ / $\mathrm{m}^{2}$ (day 1 )

Weeks $3,9,15,21$ : carboplatin $18.7 \mathrm{mg} / \mathrm{kg}$ (patients less than $10 \mathrm{~kg}$ ) or $560 \mathrm{mg} / \mathrm{m}^{2}$ (more than $10 \mathrm{~kg}$ ) (days 1 and 2), etoposide $3.3 \mathrm{mg} / \mathrm{kg}$ (less than $10 \mathrm{~kg}$ ) or $100 \mathrm{mg} / \mathrm{m}^{2}$ (more than $10 \mathrm{~kg}$ ) (days 1, 2, and 3).

No intrathecal chemotherapy was prescribed in regimens 2 and 3 . Radiotherapy guidelines were: stage Illb2: orbital radiotherapy 45 Gy up to the optic chiasm (starting at week 0 ).

Intrathecal chemotherapy included age adjusted methotrexate, cytarabine, and dexamethasone. ${ }^{6}$
The chemotherapy regimens varied over the years (table 1). Vincristine, doxorubicin, and cyclophosphamide containing regimens were the backbone of treatment before $1994^{6} 10$ (regimen 1). Since then, newer agents like carboplatin, etoposide, and idarubicin have been added to the treatment protocols $^{11}{ }^{12}$ (regimens 2 and 3). Patients with post-laminar optic nerve disease with a positive margin treated at the HPG from 1994 onwards received more intensive regimen (regimen 3).

\section{Statistical analysis}

Probability of event free survival (pEFS) and overall survival (pOS) were calculated according to Kaplan-Meier. Events were defined as extraocular relapse, death caused by tumour or associated with treatment, and second malignancies. Follow up time was calculated from the date of enucleation to the date of last contact with each patient. Survival status reflects the most recent patient contact as of 1 December 2002 .

\section{RESULTS}

\section{Patient characteristics}

Of the 224 evaluable patients, 84 were from NYPH and 140 patients were from HPG. Thirty nine additional patients were considered to be not evaluable. This was due to lack of adequate follow up information $(n=26)$. Twenty four of them were seen at our institutions only at diagnosis and were followed up elsewhere, and two cases at the HPG with cut end invasion of the optic nerve abandoned chemotherapy treatment and were lost to follow up. Nine underwent enucleation at another institution and four had overt orbital involvement at diagnosis. Table 2 summarises the histopathological findings. Median age at presentation was 24.3 months (range 1.4 to 146 months). One hundred and seven patients were male and 117 female. Two patients had a family history of retinoblastoma.

Median follow up was 49.2 months (range 12 to 242 months). Three year pEFS and pOS for the whole population were 0.95 (standard error, SE 0.001) and 0.98 (SE 0.001), respectively.

\section{Patients with no risk factors}

One hundred fifteen patients did not receive adjuvant treatment. One additional patient was treated with adjuvant chemotherapy because of previous ophthalmic surgery. No extraocular relapse was seen in any case. Included in this group were 30 patients with prelaminar or superficial optic nerve invasion alone (without concomitant choroidal or scleral invasion).

\section{Anterior chamber involvement}

Two patients had anterior chamber involvement invasion without post-laminar optic nerve or scleral involvement. They did not receive adjuvant therapy and neither suffered a

Table 2 Patient distribution according to histopathology and centre

\begin{tabular}{llll}
\hline & NYPH & HPG & Combined \\
\hline Period & $1980-2001$ & $1987-2001$ & $1980-2001$ \\
Patients (total/evaluable) & $111(84)$ & $152(140)$ & $263(224)$ \\
Intraretinal disease & 48 & 36 & 84 \\
Prelaminar optic nerve & 15 & 15 & 30 \\
Anterior chamber invasion & 1 & 1 & 2 \\
Choroidal invasion & 10 & 45 & 55 \\
Post-laminar optic nerve & 7 & 29 & 36 \\
Cut end invasion & 2 & 10 & 12 \\
Scleral invasion & 1 & 4 & 5 \\
\hline
\end{tabular}


relapse. In addition, anterior chamber invasion was present in 10 other patients with other potential risk factors described below.

\section{Isolated choroidal invasion}

There were 55 patients with isolated choroidal invasion, 16 of whom had associated prelaminar optic nerve involvement. None received adjuvant therapy. Three year pEFS is 0.94 (SE 0.003 ) and pOS is 1 . Three patients experienced extraocular relapse. One had isolated orbital relapse and the remaining two had both orbital and systemic relapse not involving the central nervous system. All received treatment for relapse; multi-agent chemotherapy and local radiotherapy for the one with isolated orbital relapse, and intensive chemotherapy followed by autologous stem cell rescue with or without external beam radiation therapy for the ones with systemic disease. All survive after treatment for extraocular relapse at 146,78 , and 16 months from relapse.

\section{Post-laminar optic nerve involvement (margin negative)}

Thirty six patients had post-laminar optic nerve disease without involvement of the cut section. Three year pEFS and pOS were 0.86 (95\% CI 0.77 to 0.95 ) and 0.91 (95\% CI 0.84 to $0.98)$, respectively. Twenty five of the 36 patients had no associated major choroidal and/or scleral invasion. Four of them received adjuvant therapy (regimen 1) while 21 did not; no patient relapsed in either group.

Eleven also had major choroidal invasion and/or scleral involvement (associated major choroidal invasion in six and both findings in five). Four of six patients with post-laminar optic nerve and major choroidal invasion did not receive adjuvant therapy and two of them relapsed (isolated orbital relapse and combined orbital and systemic relapse). Both were salvaged with chemotherapy (including high dose chemotherapy followed by autologous stem cell rescue in the one with systemic relapse) and survive disease free for 71 and 90 months from relapse. The two remaining patients with this feature received adjuvant chemotherapy and neither relapsed.

All five patients with combined post-laminar optic nerve disease and major choroidal and scleral invasion received adjuvant chemotherapy (regimen 1 in two cases and regimen 2 in three). However, three of them relapsed. The CNS was involved at relapse in all cases, being the single site of relapse in two and combined with orbital and systemic recurrence in the remaining one. An attempt to achieve a second remission was carried out in all, but no response to chemotherapy was seen and all patients died of progressive disease.

\section{Post-laminar optic nerve involvement (margin positive)}

All 12 patients with tumour at the line of section of the optic nerve received adjuvant systemic chemotherapy (regimen 1 in nine cases and regimen 3 in three) and orbital radiotherapy. Three year pEFS and pOS were 1 .

\section{Scleral invasion}

In addition to the five patients discussed in the previous section who had scleral invasion in association with postlaminar optic nerve involvement, there were five patients with scleral invasion without post-laminar optic nerve involvement. All had choroidal invasion and one had prelaminar optic nerve involvement. Adjuvant therapy was not given in two cases because of parental refusal and one had an isolated orbital relapse. She was treated with chemotherapy (regimen 3) and orbital radiotherapy and survives 62 months from relapse. The remaining three patients received adjuvant chemotherapy and one had a systemic relapse involving bone and lymph node metastases. A second remission was achieved with further chemotherapy, but he later had a second relapse and died of progressive disease.

\section{Outcome for the total group with putative high risk features}

Three year pEFS and pOS for the whole population of 108 patients with putative risk factors (choroidal invasion, scleral invasion, post-laminar optic nerve involvement, anterior chamber invasion) are 0.9 (95\% CI 0.87 to 0.93 ) and 0.96 ( $95 \%$ CI 0.95 to 0.97 ), respectively (fig 1; table 3 ).

\section{DISCUSSION}

Our philosophy of avoiding adjuvant therapy in patients with isolated choroidal, anterior chamber, and post-laminar optic nerve invasion with a negative margin without associated choroidal and/or scleral invasion, and treating intensively the few that have an extraocular relapse was associated with $100 \%$ survival. This approach allowed us to avoid the toxicity of treatment in the majority of patients who would not benefit from adjuvant chemotherapy. Patients with isolated scleral invasion, post-laminar optic nerve disease with concomitant scleral or choroidal invasion or invasion to the surgical margin are at higher risk for developing extraocular relapse and may benefit from adjuvant therapy.

By using the criteria described above, adjuvant treatment was avoided in about three quarters of the patients in our cohort. Six of them relapsed and then received intensive therapy, and all became long term survivors. We acknowledge that this policy exposed relapsed patients to the relatively high toxicity of the retrieval treatment, which included high dose chemotherapy and orbital radiation. However, given the relatively low relapse in this population, only a few very high risk patients actually received it. In contrast, all four patients who relapsed after adjuvant chemotherapy died because of metastatic disease (in the CNS in three cases and systemically in the other). Patients with CNS involvement from retinoblastoma are virtually incurable with any treatment strategy. ${ }^{6}$ Even though the chemotherapy regimens prescribed in our study included drugs with proven penetration to the CNS such as carboplatin and cyclophosphamide, they may have been insufficient for the treatment of these very high risk patients and the CNS may have acted as a sanctuary site. There is no consensus on what the best drug combination for adjuvant therapy is. We recommend that CNS directed coverage should be a major component of adjuvant chemotherapy when it is to be used.

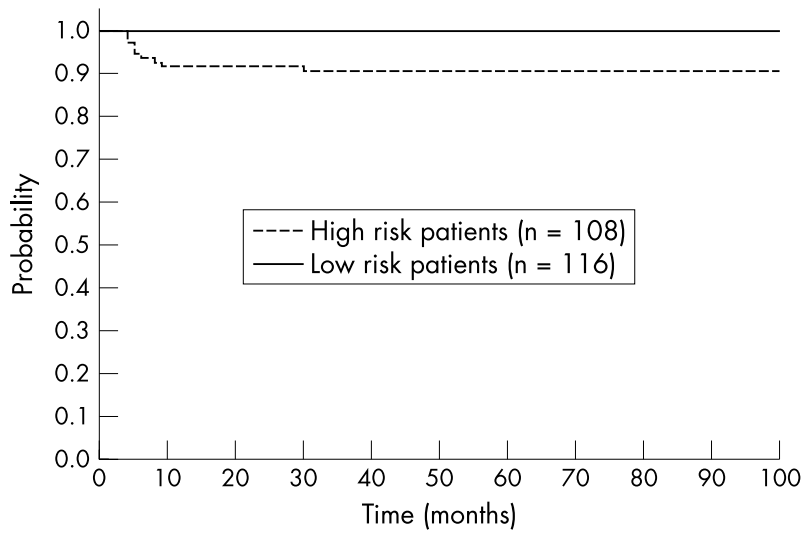

Figure 1 Probability of event free survival of 108 patients with high risk features (choroidal, scleral, anterior chamber, and post-laminar optic nerve invasion) versus 116 patients with no risk factors. 
Table 3 Treatment and outcome of patients according to risk factors detected on histopathological examination

\begin{tabular}{|c|c|c|}
\hline Feature & Adjuvant treatment & Events \\
\hline $\begin{array}{l}\text { Isolated choroidal } \\
\text { invasion }(n=55)\end{array}$ & None & $\begin{array}{l}\text { Orbital relapse }=1 ; \\
\text { orbital and systemic } \\
\text { relapse }=2\end{array}$ \\
\hline $\begin{array}{l}\text { Isolated post-laminar } \\
\text { optic nerve }(n=25)\end{array}$ & $\begin{array}{l}\text { Chemotherapy }=4 ; \\
\text { none }=21\end{array}$ & None \\
\hline $\begin{array}{l}\text { Post-laminar optic nerve } \\
\text { with major choroidal } \\
\text { invasion }(n=6)\end{array}$ & $\begin{array}{l}\text { Chemotherapy }=2 \\
\text { None }=4\end{array}$ & $\begin{array}{l}\text { None } \\
\text { Orbital and systemic } \\
\text { relapse }=1 \text {; orbital } \\
\text { relapse }=1\end{array}$ \\
\hline $\begin{array}{l}\text { Post-laminar optic nerve } \\
\text { invasion with scleral } \\
\text { invasion }(n=5)\end{array}$ & Chemotherapy $=5$ & $\begin{array}{l}\text { CNS relapse }=1 ; \text { CNS } \\
\text { and systemic } \\
\text { relapse }=2\end{array}$ \\
\hline Cut end invasion $(n=10)$ & $\begin{array}{l}\text { Chemotherapy } \\
\text { and orbital } \\
\text { radiotherapy }=10\end{array}$ & None \\
\hline $\begin{array}{l}\text { Isolated scleral invasion } \\
(\mathrm{n}=5)\end{array}$ & None $=2$ & Orbital relapse $=1$ \\
\hline $\begin{array}{l}\text { Isolated anterior } \\
\text { chamber invasion }(n=2)\end{array}$ & $\begin{array}{l}\text { Chemotherapy }=3 \\
\text { None }\end{array}$ & $\begin{array}{l}\text { Systemic relapse }=1 \\
\text { None }\end{array}$ \\
\hline
\end{tabular}

Isolated choroidal and isolated scleral invasion includes patients with less than post-laminar optic nerve involvement. Isolated post-laminar optic nerve disease includes patients with no scleral invasion or focal choroidal invasion. Isolated anterior chamber invasion included no concomitant choroidal, scleral, or post-laminar optic nerve disease.

As was previously reported, patients with only intraretinal extension and no invasion of ocular coats did well without any adjuvant therapy. ${ }^{6}{ }^{10}$ In accordance with the published literature, extraocular relapse occurred only in patients with previously identified histopathological risk features. However, this group of patients with high risk features was not homogeneous in terms of their risk for relapse.

Only two patients with isolated anterior chamber invasion were included and neither relapsed; however no definitive conclusions can be made with such a low patient number.

The management of patients with choroidal invasion is more controversial. The overall survival of all patients with any degree of choroidal invasion in our series is $100 \%$. It is clear from our study, as well as from many others, ${ }^{1-3} 56{ }^{14}$ that only a small percentage of patients with isolated choroidal invasion have an extraocular relapse and most centres report survival greater than $95 \%$ for patients with choroidal involvement. $^{236}$ Some reports suggested that only patients with massive choroidal invasion are at greater risk for extraocular relapse and therefore adjuvant chemotherapy should be prescribed in this cohort, ${ }^{1}{ }^{4}$ but other studies failed to find any difference between these subgroups in terms of risk of relapse. ${ }^{15}$ However, there is no consensus on the definition of massive invasion and not every study reports their definition of massive invasion. ${ }^{3}$ The impact of choroidal invasion as a risk factor for extraocular relapse was studied in several retrospective studies. Most authors found that massive isolated choroidal invasion was associated with an increased risk for extraocular relapse. In a retrospective study, Khelfaoui et al reported four relapses in 20 patients with massive choroidal invasion. ${ }^{1}$ However, only one of them had less than post-laminar optic nerve invasion. ${ }^{2}$ Like we have seen in our group, this child was successfully treated of relapse. ${ }^{2}$ Honavar et al recommend adjuvant chemotherapy for this subgroup based on their experience of eight patients with massive choroidal infiltration as a single risk factor, as six of their patients received adjuvant therapy and none relapsed. ${ }^{3}$ Other authors like Uusitalo et al found no relapses in their cohort of 11 patients treated only with enucleation and recommend no adjuvant therapy. ${ }^{4}$ Each group had a different definition of the extent of choroidal invasion and, as it also differed between our institutions, no attempt to separately analyse minor and massive choroidal involvement was done for this report. Given the low relapse rate in this subgroup and the fact that relapsing patients can often be rescued with intensive treatment, we prefer to withhold adjuvant chemotherapy for patients with this feature.

There is universal agreement that patients with prelaminar optic nerve disease do not need any adjuvant therapy and our results are consistent with that. There is also agreement that patients with tumour at the line of section of the optic nerve are at very high risk for relapse and they need adjuvant therapy. ${ }^{1-46}$ The results of adjuvant therapy used in our population was excellent; however it is important to note that patients with post-laminar optic nerve invasion including the cut end, who also presented with overt orbital involvement, were excluded from our analysis.

Patients with post-laminar optic nerve involvement without invasion of the cut end section of the optic nerve probably represent the most controversial group in terms of their management after enucleation. At our institutions, all patients with this feature were considered for adjuvant intravenous and intrathecal chemotherapy up to 1994. Since then, only those with associated major choroidal and/or microscopic scleral invasion received adjuvant systemic chemotherapy (without intrathecal treatment) and none of the 21 patients with isolated post-laminar optic nerve involvement with a negative margin treated with enucleation alone relapsed. In contrast, the presence of concomitant major choroidal or scleral invasion proved to be a significant risk factor, as some patients relapsed despite adjuvant chemotherapy. Khelfaoui et al found that 10 of 33 patients with post-laminar optic nerve invasion had an extraocular relapse. ${ }^{1}$ Shields et al reported that the presence of optic nerve disease alone was not associated with an increased risk of metastasis when patients with choroidal invasion in addition to post-laminar optic nerve involvement were excluded from the analysis. ${ }^{16}$ Uusitalo et al reported no relapses in 11 patients with this feature, seven of whom received adjuvant chemotherapy in their series. Their criterion for offering adjuvant treatment was based on the extension of tumour invasion along the optic nerve $(>1 \mathrm{~mm})$, rather than the presence of other risk factors as in our cohort. ${ }^{2}$ Honavar et al found that five of 29 patients with this post-laminar optic nerve involvement relapsed. Relapse was more frequent in patients who did not receive adjuvant therapy, as four of five cases of relapse occurred in patients who did not receive adjuvant therapy. ${ }^{3}$ Many authors therefore recommend adjuvant therapy for all patients with this feature. ${ }^{1-3}$ However, according to our results, two subgroups with different risks of relapse, based on the presence or absence of concomitant major choroidal or scleral involvement, can be identified.

This is the largest cohort reported in which treatment information is available thus far, and we believe that patients with concomitant choroidal or scleral involvement should be considered to be at very high risk of extraocular relapse and receive adjuvant therapy with effective CNS penetration.

There were too few patients with scleral invasion without associated post-laminar optic nerve disease to draw any definitive conclusion. Despite the lack of data, we continue to recommend adjuvant chemotherapy to patients with isolated microscopic scleral invasion.

\section{ACKNOWLEDGEMENTS}

Drs Chantada and Dunkel were supported in part by the Fund for Ophthalmic Knowledge.

\section{Authors' affiliations}

G L Chantada, Department of Hematology-Oncology, Hospital JP Garrahan, Buenos Aires, Argentina 
I J Dunkel, Department of Pediatrics, Memorial Sloan-Kettering Cancer Center, New York, NY, USA

M T G de Dávila, Department of Pathology, Hospital JP Garrahan, Buenos Aires, Argentina

D H Abramson, Department of Ophthalmology, New York Presbyterian Hospital: Weill-Cornell, New York, NY, USA

\section{REFERENCES}

1 Khelfaoui F, Validre P, Auperin A, et al. Histopathologic risk factors in retinoblastoma. A retrospective study of 172 patients treated in a single institution. Cancer 1996;77:1206-13.

2 Uusitalo M, Van Quill K, Scott I, et al. Evaluation of chemoprophylaxis in patients with unilateral retinoblastoma with high-risk features on histopathologic examination. Arch Ophthalmol 2001;119:41-8.

3 Honavar G, Singh A, Shields C, et al. Post enucleation adjuvant therapy in high risk retinoblastoma. Arch Ophthalmol 2002;120:923-31.

4 Magramm I, Abramson D, Ellsworth R. Optic nerve involvement in retinoblastoma. Ophthalmology 1989;96:217-22.

5 Stannard C, Sealy L, Sevel D. Retinoblastoma: Correlation of invasion of the optic nerve and choroid with prognosis and metastases. Br J Ophthalmol 1979;63:560-70.

6 Schvartzman E, Chantada G, Fandiño A, et al. Results of a stage-based protocol for the treatment of retinoblastoma. J Clin Oncol $1996 ; 14: 1532-6$
7 Dunkel IJ, Aledo A, Kernan NA, et al. Successful treatment of metastatic retinoblastoma. Cancer 2000;89:2117-21.

8 Namouni F, Doz F, Tanguy ML, et al. High dose chemotherapy with carboplatin, etoposide and cyclophosphamide followed by hematopoietic stem cell rescue in patients with high risk retinoblastoma: a SFOP and SFGM study. Eur J Cancer 1997;33:2368-75.

9 Chantada G, Fandiño A, Casak S, et al. Treatment of overt extraocular retinoblastoma. Med Pediatr Oncol 2003;40:158-61.

10 Grabowski E, Abramson D. Intraocular and extraocular retinoblastoma. Hematol Oncol Clin North Am 1987;1:721-35.

11 Doz F, Nevenshwander S, Plantaz D, et al. Etoposide and carboplatin in extraocular retinoblastoma: A study by the Societé Française d'Oncologie Pédiatrique. J Clin Oncol 1995;13:902-9.

12 Chantada G, Fandiño A, Mato G, et al. A phase II window of Idarubicin in children with extraocular retinoblastoma. J Clin Oncol 1999;17: 1847-50.

13 Chantada GL, de Davila MT, Fandiño A, et al. Retinoblastoma with low risk for extraocular relapse. Ophtalmic Genet 1999;20:133-40.

14 Shields C, Shields J, Baez K, et al. Choroidal invasion of retinoblastoma:metastatic potential and clinical risk factors. Br J Ophthalmol 1993;77:544-8.

15 Messmer E, Heinrich T, Hopping W, et al. Risk factors for metastases in patients with retinoblastoma. Ophthalmology 1991;98:136-41.

16 Shields C, Shields J, Baez K, et al. Optic nerve invasion of retinoblastoma. Metastatic potential and clinical risk factors. Cancer 1994;73:692-8. 